\title{
INTERSTELLAR SCATTERING TOWARD THE GALACTIC CENTER AS PROBED BY OH/IR STARS
}

\author{
Huib JaN VAN LANGEVELDE \\ Sterrewacht Leiden, Postbus 9513, 2300 RA Leiden, Netherlands \\ Dale A. Frail \\ NRAO/AOC, PO Box O, Socorro, NM 87801 \\ JAMES M. CORDES \\ Astronomy Department and N.A.I.C., Space Sciences Building, Cornell University, Ithaca, NY 14853-6801 \\ AND \\ PhILIP J. DiAmond \\ NRAO/AOC, PO Box O, Socorro, NM 87801 \\ Received 1991 November 4; accepted 1992 March 17
}

\begin{abstract}
We report here on angular broadening measurements of $20 \mathrm{OH} / \mathrm{IR}$ stars near the Galactic center. This class of sources is known to have bright, intrinsically compact ( $\leq 20$ mas) maser components within their circumstellar shells. We have used VLBA antennas and the VLA to perform a MkII spectral line VLBI experiment. The rapid drop in correlated flux with increasing baseline, especially for sources closest to the Galactic center, is attributed to interstellar scattering. Angular diameters were measured for 13 of our sources. Lower limits were obtained for the remaining seven. With our data' together with additional data taken from the literature, we have been able to determine the distribution of interstellar scattering toward the Galactic center. We find a region of pronounced scattering $\left(\bar{\theta}_{\text {obs }}=525\right.$ mas at $1.612 \mathrm{GHz}$ ) nearly centered (angularly) on Sgr $\mathrm{A}^{*}$. We consider two interpretations for the enhanced scattering. One hypothesis is that the scattering is due to a clump of enhanced turbulence, such as those that lie along lines of sight to other known objects, that has no physical relationship to the Galactic center. The other model considers the location of the enhanced scattering to arise in the Galactic center itself. The physical implications of the models yield information on the nature of the interstellar scattering. Future tests to discriminate between these models will be the measurement of angular sizes of extragalactic sources within $15^{\prime}$ of Sgr $\mathrm{A}^{*}$ and angular sizes of additional OH/IR stars toward the Galactic center region.
\end{abstract}

Subject headings: circumstellar matter - masers - techniques: interferometric

\section{INTRODUCTION}

It has long been suspected that the observed size of the Galactic center source Sgr A* is set by interstellar scattering (Davis, Walsh, \& Booth 1976; Lo et al. 1981; Lo et al. 1985; Backer 1988). At $1.6 \mathrm{GHz}$, the frequency we use throughout this paper, the scattered broadened diameter $\theta_{\text {obs }}$ of $\operatorname{Sgr} \mathrm{A}^{*}$ is about 520 mas. Such a high value of intestellar scattering is unusual, as there are only a few lines of sight with comparable degrees of plasma turbulence (Cordes, Ananthakrishnan, \& Dennison 1984; Moran et al. 1990; Fey, Sprangler, \& Cordes 1991). Is this scattering anomalous, local to Sgr $\mathrm{A}^{*}$ and a consequence of the activity occurring there (Backer 1988)? Is it due to clumps of strong turbulence at some random position along the line of sight (Cordes, Weisberg, \& Boriakoff 1985)? Or can it be explained as part of the general increase in scattering that is observed toward the inner Galaxy (Fey et al. 1991; Rao \& Slee 1988)? The answer to this question has a bearing on an unsolved problem in the study of plasma turbulence in the interstellar medium: the identity of the agent(s) responsible for the turbulence.

Recently, Van Langevelde \& Diamond (1991) showed that the $\mathrm{OH}$ masers in the circumstellar shells of four OH/IR stars, all less than $16^{\prime}$ from $\mathrm{Sgr} \mathrm{A}^{*}$, were broadened by an amount similar to Sgr A*. Thus they confirmed that the size of Sgr A* at centimeter wavelengths is set by interstellar scattering. An equally important consequence of this work was the realiza- tion that $\mathrm{OH} / \mathrm{IR}$ stars are a powerful tool to study the turbulent electron density component in the very inner regions of our Galaxy. The number of observable $\mathrm{OH} / \mathrm{IR}$ stars in this region is at least an order of magnitude larger than that of extragalactic sources, and they are all at roughly similar distances. Moreover, as we will show in $\S 2$, there exist bright, intrinsically compact ( $<20$ mas) maser spots in all $\mathrm{OH} / \mathrm{IR}$ stars and so the usual problem of intrinsic source structure for extragalactic sources does not enter into the interpretation of the visibility data. Pulsars, which in general are excellent point source probes on interstellar scattering, cannot be used to obtain information on interstellar scattering close to the Galactic center. Although pulsars are probably present within a few kpc of the Galactic center (e.g., Clifton \& Lyne 1986), it is unlikely that they will be found within $100 \mathrm{pc}$, because interstellar scattering will quench the pulsations.

In this paper we expand on the earlier work of Van Langevelde \& Diamond (1991) with the goal of determining the distribution of the scattering material around $\mathrm{Sgr} \mathrm{A}^{*}$ in order to place constraints on the properties and the location of this medium. The outline of this paper is as follows. We start by describing our observational technique as it applies to the angular broadening of $\mathrm{OH} / \mathrm{IR}$ stars specifically. This includes setting up the necessary formulas to describe scattering, as well as the relation between interstellar scattering and the emission measure, which we will use to discuss the possibility of a scat- 
tering medium located at the Galactic center. In $\S 3$ we discuss our source selection criteria, the observational parameters, and the reduction of the visibility data to scatter diameters. Finally, in $\S 4$, we use these diameters, in addition to those from the literature, to examine possible models for the source of scattering in the direction of the Galactic center.

\section{TECHNIQUE}

\subsection{Angular Broadening by Interstellar Scattering}

Angular broadening has its origin in fluctuations of the electron density in the interstellar medium. Because the refraction index of the interstellar medium for radio waves is given by $n \approx 1-\left(r_{e} n_{e} \lambda^{2} / 2 \pi\right)$, fluctuations in $n_{e}$ cause disturbances in a wave front traveling through the interstellar medium. This degrades the degree of coherence between the signals received at two widely separated points, by an amount that depends on the level of turbulence present in the medium. Electron density fluctuations on scales of $10^{6}-10^{8} \mathrm{~m}$ are readily measurable with an interferometer by determining either the loss of coherence as a function of antenna separation (in the visibility plane) or, equivalently, the angular broadening (in the image plane).

The same fluctuations give rise to a variety of other observational phenomena (see Rickett 1990 for a review), but we will concern ourselves in this paper with angular broadening alone. Following other authors in the study of the Galactic distribution of scattering material, we will make some simplifying assumptions. Apart from the fact that these assumptions are readily defendable, they also enable us to compare easily our results with others.

\subsubsection{The Power-Law Description}

Following Cordes et al. (1985), we describe fluctuations in the electron density by an isotropic power law:

$$
P_{\delta n_{e}}(q, x)=C_{n}^{2}(x) q^{-\alpha}
$$

which holds between lower and upper wavenumber cutoffs: $q_{0} \leq q \leq q_{1}$ and where $x$ denotes the line of sight from the source to the observer. $C_{n}^{2}(x)$ is the power in the fluctuation spectrum at some position along the line of sight [the changes in $C_{n}^{2}(x)$ take place on a much larger scale than the electron density fluctuations]. We are here particularly interested in the theory appropriate for the source close to the scattering medium.

There have been several studies specifically to determine $\alpha$, $q_{0}$, and $q_{1}$ (Armstrong, Cordes, \& Rickett 1981; Cordes et al. 1985; Clegg \& Simonetti 1990a; Gwinn et al. 1988; Spangler \& Gwinn 1990). Determining these values is beyond the scope of our observations, and so we use $\alpha=11 / 3$ throughout this paper. This is a defendable choice and allows us to compare our results directly with other measurements of the distribution of interstellar scattering.

The power spectrum above will produce a phase structure term in a propagating wave through the medium. This will affect the measured visibility of a point source at a distance $L$, which is described by

$$
V(\rho, L)=\exp \left[-D_{\phi}(\rho) / 2\right]
$$

where $\rho$ is the baseline length and $D_{\phi}(\rho)$, the phase structure function, is given by

$$
D_{\phi}(\rho)=8 \pi^{2} r_{e}^{2} \lambda^{2} \int_{0}^{L} \int q\left[1-J_{0}(q \rho)\right] P_{\delta n_{e}}(q, x) d q d x
$$

for the case of a plane wave (source at infinity). For a source embedded in the medium it has been shown (Ishimaru 1978, p. $418)$ that $J_{0}(q \rho)$ is replaced by $J_{0}(q \rho x / L)$, where $(x / L)$ is a dilution factor. For $2 \pi / q_{1} \ll \rho \ll 2 \pi / q_{0}$, this results in

$$
V(\rho, L)=\exp \left[-\left(\rho / \rho_{c}\right)^{\alpha-2}\right]
$$

where

$$
\rho_{c}=\left[4 \pi^{2} \lambda^{2} r_{e}^{2} \mathscr{S}(L) f(\alpha)\right]^{-1 /(\alpha-2)}
$$

in which $f(\alpha)$ is an integration constant equal to 1.11835 for $\alpha=11 / 3 . \mathscr{S}(L)$ takes into account the integration of $C_{n}^{2}(x)$ from source to observer. It equals

$$
\mathscr{S}(L)=\int_{0}^{L} C_{n}^{2}(x)\left(\frac{x}{L}\right)^{\alpha-2} d x
$$

where the observed source lies within the medium, or

$$
\mathscr{S}(L)=\int_{0}^{L} C_{n}^{2}(x) d x
$$

when observing an extragalactic source. In this last case $\mathscr{S}(L)$ is by definition the scattering measure, SM. This quantity is directly usable in the case of an extragalactic source. For a source embedded in the medium, as in this work, an additional assumption is needed before the SM can be used. If we make $C_{n}^{2}(x)$ constant, equation (5) can be expressed as $\mathscr{S}(L)=(\alpha-1)^{-1} \mathrm{SM}$ (Cordes et al. 1991a). If $C_{n}^{2}$ is not a constant then equation (5) should be used. Note that there is a strong weighting of scattering along the line of sight. The effect is that electron turbulence close to the source is weighted much less strongly $\left[\propto(x / L)^{\alpha-2}\right]$ than material more distant from the source.

\subsubsection{The Very Strong Scattering Regime}

The analysis embodied in equations (3)-(5) assumes that the relevant baselines (i.e., $\rho \approx \rho_{c}$ ) are bracketed by the inner and outer scales of the electron density power spectrum:

$$
l_{1} \equiv 2 \pi q_{1}^{-1} \ll \rho \ll l_{0} \equiv 2 \pi q_{0}^{-1} .
$$

In very strong scattering, the visibility function becomes narrower than the inner scale, $l_{1}$. In this case, different expressions for the scattering measure hold. Other researchers (e.g., Spangler \& Gwinn 1990; Moran et al. 1990; Molnar et al. 1990) have found evidence in favor of an inner scale $l_{1} \approx 100 \mathrm{~km}$, suggesting that any measured angular diameters $\theta_{\text {obs }}$ larger than about 100 mas at $1 \mathrm{GHz}$ are influenced by finite inner scale effects.

There are several consequences of a finite inner scale which should be mentioned. First, very strong scattering that yields a very small spatial scale for the visibility does not require correspondingly small scale sizes in the scattering medium. Second, in very strong scattering, the visibility function is a Gaussian and the scattering diameter scales as $\theta_{\text {obs }} \propto \lambda^{2}$. Thus

$$
V(\rho, L)=\exp \left[-\left(\rho / \rho_{c}\right)^{2}\right] \quad \rho<l_{1}
$$

where it can be shown (e.g., Cordes \& Lazio 1991) that

$$
\rho_{c}=\left[2 \pi^{2} \lambda^{2} r_{e}^{2} \mathscr{P}(L) q_{1}^{4-\alpha}(4-\alpha)^{-1}\right]^{-1 / 2} .
$$

Third, the line-of-sight weighting is changed slightly, such that, for sources embedded in the medium, equation (5) is replaced with

$$
\mathscr{S}(L)=\int_{0}^{L} C_{n}^{2}(x)\left(\frac{x}{L}\right)^{2} d x
$$

The result of equation (6) still holds for extragalactic sources. 


\subsubsection{Resulting Expressions}

In general we give the following results from equation (9) and (4), respectively, expressed in observable quantities. In particular we have expressed the transition to the strong scattering regime by $\theta_{\text {cross }}$; for scatter sizes larger than this value, the formulae derived for the strong scattering regime hold:

$$
\mathscr{S}(L)= \begin{cases}\left(\frac{\theta_{\mathrm{obs}}}{133 \mathrm{mas}}\right)^{2}\left(\frac{l_{1}}{100 \mathrm{~km}}\right)^{1 / 3} v_{\mathrm{GHz}}^{4}, & \theta_{\mathrm{obs}} \geq \theta_{\text {cross }} \\ \left(\frac{\theta_{\mathrm{obs}}}{128 \mathrm{mas}}\right)^{5 / 3} v_{\mathrm{GHz}}^{11 / 3}, & \theta_{\text {obs }} \leq \theta_{\text {cross }}\end{cases}
$$

where $\theta_{\text {cross }} \approx 0.16^{\prime \prime}\left[v_{\mathrm{GHz}}\left(l_{1} / 100 \mathrm{~km}\right)\right]^{-1}$.

To give the reader some idea about the numbers involved, we mention here that the values of $C_{n}^{2}(x)$ determined for the solar neighborhood are $\sim 10^{-3.5} \mathrm{~m}^{-20 / 3}$, resulting in an angular size of about 1 mas for an extragalactic source at $18 \mathrm{~cm}$ for a line of sight of $1 \mathrm{kpc}$ through the medium. $C_{n}^{2}$ is typically 1 $\mathrm{m}^{-20 / 3}$ in the inner Galaxy resulting in up to 150 mas for objects seen through the inner Galaxy.

\subsubsection{Electron Densities and Emission Measures}

The electron density variations responsible for scattering also contribute to free-free radiation. The calculation of the emission measure EM involves $n_{e}=\bar{n}_{e}+\delta n_{e}$ with $\bar{n}_{e}$ the local mean density and $\delta n_{e}$ the local variation about the mean. To estimate the rms electron density $\delta n_{e}$ one integrates $P_{\delta n_{e}}$, the fluctuation spectrum. For $\alpha=11 / 3$ and an outer scale $l_{0} \gg l_{1}$,

$$
\begin{aligned}
\delta n_{e}(\mathrm{rms})=\left(6 \pi C_{n}^{2}\right)^{1 / 2}\left(\frac{l_{0}}{2 \pi}\right)^{1 / 3} & \\
& \approx 0.03 \mathrm{~cm}^{-3}\left(\frac{C_{n}^{2}}{10^{-3} \mathrm{~m}^{-20 / 3}}\right)^{1 / 2}\left(\frac{l_{0}}{1 \mathrm{pc}}\right)^{1 / 3} .
\end{aligned}
$$

This will give a contribution to the EM, which may be written as

$$
\begin{aligned}
\mathrm{EM}_{\mathrm{SM}} \approx \frac{2(2 \pi)^{1 / 3}}{2 / 3} l_{0}^{2 / 3} \mathrm{SM} & \\
& =10^{2.74} \mathrm{pc} \mathrm{cm}^{-6}\left(\frac{l_{0}}{1 \mathrm{pc}}\right)^{2 / 3}\left(\frac{\mathrm{SM}}{1 \mathrm{kpc} \mathrm{m}^{-20 / 3}}\right)
\end{aligned}
$$

(Cordes et al. 1991a, b). This corresponds to a minimum optical depth

$$
\tau_{\mathrm{ff}, \mathrm{SM}}=\frac{1}{10^{3.54}}\left(\frac{\mathrm{SM}}{1 \mathrm{kpc} \mathrm{m}^{-20 / 3}}\right)\left(\frac{l_{0}}{1 \mathrm{pc}}\right)^{2 / 3} \frac{g(v, T)}{v_{\mathrm{GHz}}^{2} T_{4}^{1.5}},
$$

where $T_{4}=\left(T / 10^{4} \quad \mathrm{~K}\right)$ and $g(v, T)=1+0.085 \ln T_{4}+$ $0.387 \ln v_{\mathrm{GHz}}$. Density fluctuations are bounded, locally, by $\delta n_{e} \leq \bar{n}_{e}$, so the actual EM and optical depth are at least a factor of 2 larger than the values in equation (13) and equation (14).

\section{2. $\mathrm{OH} / \mathrm{IR}$ Stars}

In order to fully appreciate why we can use OH/IR stars to study interstellar scattering, we must first discuss the properties of $\mathrm{OH}$ masers in circumstellar shells. From the moment of their discovery, it was suggested that the strong $1612 \mathrm{MHz}$ spectral lines with their typical two-peaked signature are caused by maser emission in expanding circumstellar shells
Wilson \& Barett 1968). In the last two decades several aspects of these $\mathrm{OH} / \mathrm{IR}$ stars have become clear. First, we now know that the underlying stars are objects on the asymptotic giant branch. Having reached their final stages of evolution, these stars are losing a considerable percentage of their mass ( $\left.\leq 10^{-4} M_{\odot} \mathrm{yr}^{-1}\right)$, resulting in a circumstellar shell which is gradually flowing away from the star. Dust condenses in this shell and is responsible for the prominent IR signature of these stars. Because of the large, cool, optically thick dust shell we see all the luminosity of the giant star being emitted longward of $5 \mu \mathrm{m}$.

This IR emission causes a population inversion in the $\mathrm{OH}$ molecules, which are abundant in the shell. Provided that long coherent pathlengths are available, line emission in such a medium can develop into a maser. Precisely because the shell is expanding, the maser works most efficiently along the radial lines in the shell. Here all $\mathrm{OH}$ molecules in the shell can "see" one another; in more tangential lines the different parts of the shell cannot amplify each other's emission because of a relative Doppler shift. Therefore every observer in any direction from the star sees the brightest emission from the extreme back and front of the shell, the outermost blue- and redshifted parts of the spectrum.

This maser beaming, arising from the fact that the shell is expanding, results in a higher brightness temperature for the peaks of the spectrum than for the inner parts.

From interferometric observations it is clear that the brightness temperatures of the inner parts of the spectrum are typically of the order of $10^{4} \mathrm{~K}$, while for the peaks of the spectrum this can be at least $10^{8} \mathrm{~K}$ (see Alcock \& Ross 1988, and references therein). In several cases (Norris et al. 1984; Benson \& Mutel 1979; Bowers et al. 1980; Reid et al. 1977) hot spots with angular sizes of the order of 10 mas are detected which have corresponding $10^{9}-10^{10} \mathrm{~K}$ brightness temperatures.

\section{3. $\mathrm{OH} / \mathrm{IR}$ Stars as Probes of Interstellar Scattering}

In this paper we interpret measurements of the angular sizes of the peak maser emission from $\mathrm{OH} / \mathrm{IR}$ stars as scatter broadening caused by interstellar scattering. Of course we can do this only when we can safely rule out that the intrinsic angular brightness distribution has no effect on our measurements, treating the underlying $\mathrm{OH}$ masers as point sources. For most sources in the survey we present here, we detect brightness temperatures of just $10^{6} \mathrm{~K}$ for the peak of the spectrum, which we safely attribute to interstellar scattering. In this respect it is interesting to discuss the result by Diamond et al. (1988), who show that the angular sizes of the peaks in OH/IR stars increase with distance. This result indicates that interstellar scattering determines the sizes (and brightness temperatures) for $\mathrm{OH}$ masers at large distances, as had been suspected earlier (Burke et al. 1968; Boyd \& Werner 1972; Moran et al. 1973; Bowers et al. 1980; Kent \& Mutel 1982; Gwinn et al. 1988; Kemball, Diamond, \& Montovani 1988).

Because all these authors fail to find sources with angular sizes less than 20 mas more distant then $1 \mathrm{kpc}$, there is good reason to believe that the maximum brightness temperature measurements for $\mathrm{OH}$ masers are frequently limited by interstellar scattering. With this in mind, we feel safe to use all measurements of the angular size above 50 mas as a measurement of interstellar scattering for sources at the distance to the Galactic center. This might also be valid for sources with smaller sizes, but to be conservative these should be interpreted as upper limits of angular broadening. 


\section{OBSERVATIONS}

\subsection{Source Selection}

In order to map the distribution of interstellar scattering about Sgr A*, we require a large sample of bright $\mathrm{OH} / \mathrm{IR}$ stars. Fortunately, there is a strong concentration of OH/IR stars with $1^{\circ}$ of the Galactic center (Lindqvist et al. 1992). Approximately half of the $20 \mathrm{OH} / \mathrm{IR}$ stars used in our work (see Table 1) were taken from the Lindqvist et al. (1992) survey in which 134 sources were discovered in the central $1.5 \mathrm{deg}^{2}$ of our Galaxy. Since the $\mathrm{VLA}^{1}$ was used for their survey, subarcsecond positions were already available for our work. For sources that have $|b|>0.5$, the all-sky survey of te Lintel Hekkert et al. (1991) was used instead. The best positional information in the te Lintel Hekkert et al. (1991) survey was based upon a match between the OH/IR star (detected at 1612 $\mathrm{MHz}$ ) and its nearest IRAS point source with the proper colors. These positions were only accurate to $\pm 15^{\prime \prime}$, and so a short VLA astrometry program had to be carried out before the VBLI observations could be done.

\subsection{Astrometry}

On 1990 June 21, the VLA was used in the hybrid A/B array configuration and two polarizations of 64 spectral line channels each were recorded across a bandwidth of $391 \mathrm{kHz}$ (or 6.1 $\mathrm{kHz}$ per channel). The data were reduced within the AIPS package following standard practice. Table 1 contains the results of this work along with the positions of the lower lati-

\footnotetext{
1 The Very Large Array (VLA) and the Very Long Baseline Array (VLBA) are operated by the National Radio Astronomy Observatory under cooperative agreement with the National Science Foundation.
}

tude sources from the Lindqvist et al. (1992) catalog. Column (1) lists the source name given with a preface of "OH," followed by its Galactic coordinates, while columns (2) and (3) are the right ascension and declination of each source, respectively. Columns (4) and (5) give the velocities for the blue and redshifted peaks of the double-peaked profiles. Similarly, the peak flux densities for these components are given in columns (5) and (6). Because OH/IR stars are known to be highly variable these values should only be taken as approximate.

We observed $15 \mathrm{OH} / \mathrm{IR}$ stars from the catalog by te Lintel Hekkert et al. (1991); 13 were detected. 17338-2744 and $17392-2722$ were not detected. For all but one of the 13 detected sources there is an IRAS source name in column (8). $\mathrm{OH} 0.334-0.181$ was paired with $17439-2845$ by te Lintel Hekkert et al. (1991), but at our new VLA position, 1.5 away, there is no known $I R A S$ source. This is likely due to confusion in the IRAS data, because of the low latitude. Another three $\mathrm{OH} / \mathrm{IR}$ stars of these 13 were found to be associated with an $I R A S$ point source other than the one that te Lintel Hekkert et al. (1991) assigned. The original IRAS names of these sources are found just below Table 1. Apart from these six cases we believe the other IRAS assignments to be correct. The poor detection statistics should not be taken as typical for the te Lintel Hekkert et al. (1991) survey, because the stellar density is very high in this region. There are no $I R A S$ designations for the Lindquist et al. (1992) sources in Table 1, owing to the severe confusion that occurs in the infrared at these lower latitudes.

\subsection{The Distance Problem}

The selection of appropriate $\mathrm{OH} / \mathrm{IR}$ stars from these catalogs was governed primarily by considerations of sensitivity. In

TABLE 1

LIST OF OBSERVED OH/IR STARS

\begin{tabular}{|c|c|c|c|c|c|c|c|}
\hline \multirow[b]{2}{*}{$\begin{array}{c}\text { Source } \\
\text { NAME } \\
(1)\end{array}$} & \multirow[b]{2}{*}{$\begin{array}{l}\text { R.A. (1950) } \\
\text { (2) }\end{array}$} & \multirow[b]{2}{*}{$\begin{array}{c}\text { DECL. (1950) } \\
\text { (3) }\end{array}$} & \multicolumn{2}{|c|}{ Peak Velocities } & \multicolumn{2}{|c|}{ Peak Fluxes } & \multirow[b]{2}{*}{$\begin{array}{c}\text { IRAS } \\
\text { DESIGNATION } \\
(8)\end{array}$} \\
\hline & & & $\begin{array}{c}\text { Blueshifted } \\
\left(\mathrm{km} \mathrm{s}^{-1}\right) \\
(4)\end{array}$ & $\begin{array}{c}\text { Redshifted } \\
\left(\mathrm{km} \mathrm{s}^{-1}\right) \\
(5)\end{array}$ & $\begin{array}{c}\text { Blueshifted } \\
\text { (Jy) } \\
\text { (6) }\end{array}$ & $\begin{array}{c}\text { Redshifted } \\
\text { (Jy) } \\
\text { (7) }\end{array}$ & \\
\hline OH $357.849+9.744$. & $17^{\mathrm{h}} 00^{\mathrm{m}} 49^{\mathrm{s}} .34$ & $-25^{\circ} 12^{\prime} 23^{\prime \prime} .3$ & -31.6 & -11.2 & 1.7 & 0.5 & $17008-2512$ \\
\hline OH $0.125+5.111 \ldots$ & 172322.90 & -260224.1 & -151.4 & -135.6 & 2.3 & 1.1 & $17233-2602$ \\
\hline OH $353.298-1.537 \ldots \ldots$ & $1731 \quad 36.51$ & -352355.9 & 48.5 & 74.6 & 3.1 & 3.6 & $17316-3523$ \\
\hline OH $359.140+1.137 \ldots \ldots$ & $1735 \quad 56.95$ & -290224.7 & -145.7 & -127.5 & 2.4 & 1.8 & $17359-2902$ \\
\hline OH $359.564+1.287 \ldots \ldots$ & $1736 \quad 24.70$ & -283610.0 & 17.9 & 45.2 & 1.7 & 0.5 & $17364-2836^{a}$ \\
\hline OH $355.641-1.742 \ldots \ldots$ & $1738 \quad 34.34$ & -333213.9 & -245.7 & -224.2 & 3.3 & 5.1 & $17385-3332$ \\
\hline OH $355.897-1.754 \ldots \ldots$ & 173916.11 & -331916.1 & -58.0 & -30.8 & 1.1 & 1.4 & $17392-3319$ \\
\hline OH $0.892+1.342 \ldots \ldots \ldots$ & 173924.74 & -272702.0 & -123.3 & -93.7 & 49.0 & 0.8 & $17393-2727$ \\
\hline OH $1.212+1.258 \ldots \ldots \ldots$ & 174029.90 & -271325.3 & 30.4 & 57.7 & 5.8 & 4.9 & $17404-2713$ \\
\hline OH $359.517+0.001 \ldots \ldots$ & 174116.555 & -291935.19 & -145.6 & -111.5 & 0.9 & 1.5 & \\
\hline OH $359.762+0.120 \ldots \ldots$ & 174124.132 & -290320.94 & -21.0 & 9.6 & 1.8 & 3.3 & \\
\hline $\mathrm{OH} 1.369+1.003 \ldots \ldots \ldots$ & 174150.64 & -271330.4 & -27.4 & 3.3 & 8.7 & 6.8 & $17418-2713$ \\
\hline OH $359.581-0.240 \ldots \ldots$ & $1742 \quad 22.155$ & -292356.71 & -103.6 & -75.2 & 3.1 & 0.9 & \\
\hline OH $359.880-0.087 \ldots \ldots$ & $1742 \quad 29.643$ & -290353.70 & -34.6 & -14.2 & 6.6 & 2.0 & \\
\hline OH $359.986-0.061 \ldots \ldots$ & 174238.757 & -285740.35 & -7.4 & 50.5 & 1.2 & 1.0 & \\
\hline OH $0.319-0.040 \ldots \ldots .$. & $1743 \quad 21.902$ & -283958.32 & 57.3 & 92.2 & 1.3 & 3.4 & \\
\hline OH $0.814+0.179 \ldots \ldots \ldots$ & 174341.639 & -280747.81 & 56.2 & 88.8 & 0.5 & 1.9 & \\
\hline OH $0.334-0.181 \ldots \ldots \ldots$ & 174356.62 & -284339.1 & -356.4 & -326.9 & 3.5 & 2.9 & \\
\hline OH $5.026+1.491 \ldots \ldots \ldots$ & $1748 \quad 26.09$ & -235056.3 & 106.7 & 135.1 & 3.0 & 1.2 & $17484-2350^{b}$ \\
\hline OH $3.234-2.404 \ldots \ldots \ldots$ & 175918.87 & -272158.8 & -45.2 & -20.2 & 4.6 & 0.2 & $17593-2721^{\mathrm{c}}$ \\
\hline
\end{tabular}

a $17363-2833$.

b $17486-2345$.

c $17596-2716$. 
order to make a detection within the coherence time on a VLA-VLBA baseline with the characteristically small linewidths of these masers $(\sim 6 \mathrm{kHz})$, we must select stars with a peak flux above $1.5 \mathrm{Jy}$. This condition was relaxed somewhat in a few instances along interesting lines of sight. In addition, as a check, we included one of the sources from Van Langevelde \& Diamond (1991).

To simplify any comparison of the scattering properties between these different lines of sight, it is important to choose the sample of $\mathrm{OH} / \mathrm{IR}$ stars so that they are located at or beyond the Galactic center. From their flattened distribution and kinematics we know, at least in a statistical sense, that the Lindqvist et al. (1992) survey contains stars at the Galactic center. For the te Lintel Hekkert et al. (1991) sample, the situation is a bit less certain because the density contrast toward the center $\theta_{\text {obs }}$ of the Galaxy is less pronounced than in the Lindqvist et al. (1992) sample. Fortunately, the IRAS data yield a useful discriminant. While the $1612 \mathrm{MHz}$ maser emission can vary widely from one star to the next (Gaylard et al. 1989; Röttgering 1989; Dickinson 1987), the variation in bolometric stellar luminosity is relatively more well behaved. This enables us to obtain some distance information about individual objects.

Thus, obvious foreground stars can be eliminated from our sample by choosing only those stars whose IRAS fluxes are weak. We have estimated a distance from the IRAS 12 and 25 $\mu \mathrm{m}$ bands using a standard luminosity of $15,000 L_{\odot}$, which is a reasonable estimate of the luminosity of these OH-emitting AGB stars (e.g., Van Langevelde, Van der Heiden \& Van Schooneveld). For this estimate we have used the bolometric correction for OH/IR stars given by Van der Veen \& Breukers (1989). We eliminated all sources whose distances calculated in this manner were less than $8 \mathrm{kpc}$. Work to derive the IR fluxes for the low latitude sources in Lindqvist et al. (1992) is in progress (Blommaert et al. 1992).

\subsection{VLBI Observations}

The observations were made on two separate occasions, 1990 October 18 and 1991 February 1. The phased VLA and all the working antennas of the VLBA were used in both cases. This included antennas at Pie Town (PT), Los Alamos (LA), Kitt Peak (KP), and Fort Davis (FD). The point source calibrator $1748-253$ was observed for several minutes each halfhour to re-phase the elements of the VLA, and the bright source NRAO 530 was observed every $4 \mathrm{hr}$ to act as a "fringe finder" during correlation. Each $\mathrm{OH} / \mathrm{IR}$ star was observed for 10-12 minutes at a time, with two scans made at different hour angles so as to sample a full range of baseline lengths.

The data were recorded on MkII tapes with a total bandwidth of $500 \mathrm{kHz}$. The tapes were processed on the NRAO MkII correlator, splitting the bandwidth into 96 spectral line channels of $5.2 \mathrm{kHz}$ each $\left(0.97 \mathrm{~km} \mathrm{~s}^{-1}\right)$. After correlation the data were further processed in AIPS following procedures outlined by Diamond (1989). The bandpass correction was made using the total power spectrum from each antenna for NRAO 530 and the data were corrected for Doppler tracking. In the first session, the amplitude calibration was done in the same manner as explained in Van Langevelde \& Diamond (1991) by using the monitored system temperatures and estimated antenna gain factors, in much the same way as is done with continuum VLBI. In the second session, however, the VLA data showed signs of severe broadband contamination, most likely from the GLONASS network of satellites. A full ampli- tude calibration was impossible. Instead we either estimated calibration constants from a priori information, or used only VLA-VLBA baselines for further analysis. Because all VLBA antennas are essentially identical, we expected VLA-VLBA baselines to be equally sensitive within $10 \%$, as was found during the first run.

A fringe fit was done for our calibrator, NRAO 530. We also had observations of $1748-253$ every half-hour to rephase the VLA, so we were able to use it to fringe fit. In this way we obtained reasonable solutions for delay and fringe rates for all antennas during the entire observation period. We then continued with a fit of the residual fringe rate on the $\mathrm{OH}$ maser sources, setting a window on the spectral channels in which we expected the peak fluxes. We set the threshold for an acceptable detection to $4 \sigma$, where we had limited the search for a fringe to the spectral channel where we expected a signal. By performing a residual fringe fit we gave up the absolute position information on the $\mathrm{OH}$ source.

In principle, the final step in obtaining the results we are interested in is straightforward. We have the spectrum of the source from a number of baselines and a "zero spacing" measurement from the autocorrelation spectrum on any of the telescopes. The autocorrelation spectrum on the VLA is most often used for the latter, because it has the highest signal-tonoise and benefits from the lower confusion provided by its small synthesized beam. At this point we simply fit a Gaussian profile to the visibility data of the brightest channel. We determine $\theta_{\text {obs }}$, the full width at half-maximum of the corresponding Gaussian in the map plane, directly from this value.

Our basic data set and fitting procedure is illustrated in Figure 1 where we show the results for $\mathrm{OH} 0.125+5.111$. Although this is a relatively weak source, it was detected on all the baselines present (PT-LA is missing, because of a technical

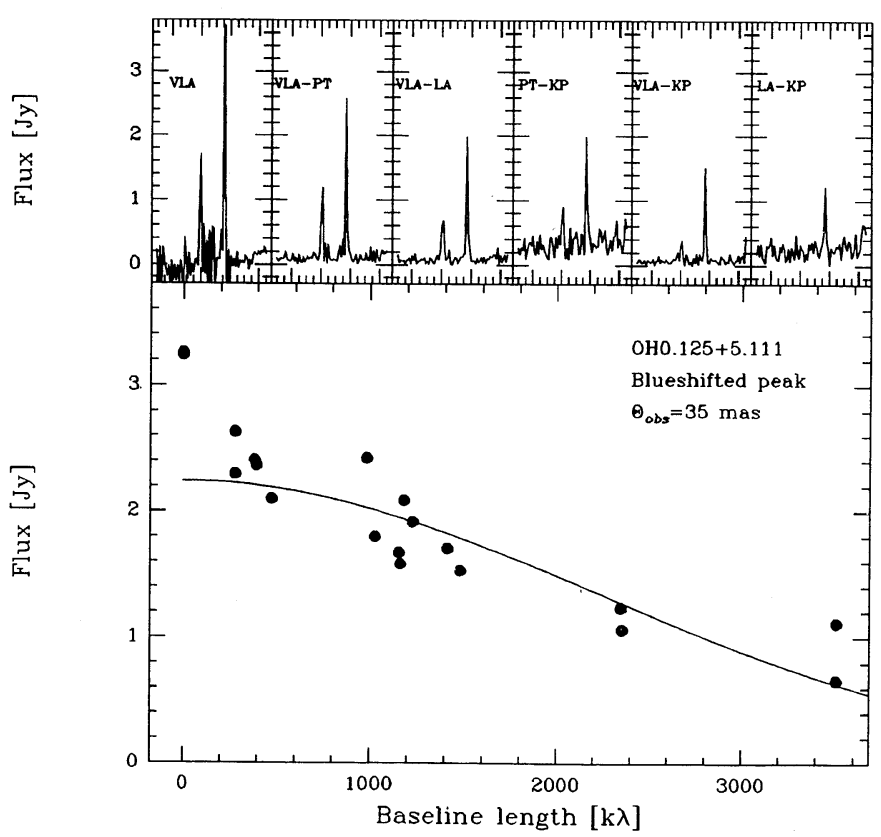

Fig. 1.-The amplitude of $\mathrm{OH} 0.125+5.111$ as a function of baseline. The top row shows the spectra as detected on baselines of increasing length toward the right. The bottom shows the amplitude of the brightest, blueshifted peak as a function of baseline length together with the fitted model for a spherical Gaussian. Because we assume a single component and the data have been subjected to fringe fitting, we disregard the phase information and take it to be zero on average. 
TABLE 2

SCATtering Diameter MEasurements of OH/IR Stars at $1612 \mathrm{MHz}$

\begin{tabular}{|c|c|c|c|}
\hline $\begin{array}{l}\text { Source } \\
\text { Name } \\
\text { (1) }\end{array}$ & $\begin{array}{c}\theta_{\text {obs }} \\
\text { (mas) } \\
(2)\end{array}$ & 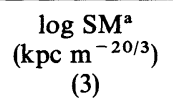 & $\Delta \underset{\text { (4) }}{\operatorname{Sgr}} A^{*}$ \\
\hline OH $353.298-1.537 \ldots$ & 360 & 2.17 & $408^{\prime} 6$ \\
\hline OH $355.641-1.742 \ldots \ldots \ldots \ldots$ & 329 & 2.09 & 277.5 \\
\hline OH $355.897-1.754 \ldots \ldots \ldots \ldots$ & 348 & 2.14 & 263.5 \\
\hline OH $357.849+9.744 \ldots \ldots \ldots \ldots$ & 157 & 1.45 & 600.6 \\
\hline OH $359.140+1.137 \ldots \ldots \ldots \ldots$ & 269 & 1.92 & 85.8 \\
\hline OH $359.564+1.287$ & 81 & 0.86 & 83.2 \\
\hline OH $359.762+0.120$ & 495 & 2.45 & 14.8 \\
\hline OH $0.125+5.111 \ldots \ldots \ldots \ldots \ldots$ & 35 & 0.25 & 309.6 \\
\hline OH $0.892+1.342 \ldots \ldots \ldots \ldots \ldots$ & 57 & 0.60 & 100.9 \\
\hline OH $1.212+1.258 \ldots \ldots \ldots \ldots \ldots$ & 258 & 1.88 & 109.1 \\
\hline OH $1.369+1.003 \ldots$ & 112 & 1.16 & 106.2 \\
\hline OH $3.234-2.404 \ldots \ldots \ldots \ldots \ldots$ & 141 & 1.36 & 242.8 \\
\hline OH $5.026+1.491 \ldots \ldots \ldots \ldots \ldots$ & 68 & 0.73 & 318.5 \\
\hline
\end{tabular}

a Assuming a medium with uniform $C_{n}^{2}$ and $l_{1}=100 \mathrm{~km}$.

problem). Note that this particular source is not heavily scattered, while for most of the sources in our sample, detections were made on only the shortest and most sensitive baselines.

In Table 2 we list the measured angular sizes $\theta_{\text {obs }}$ of all sources that were detected on one or more baselines. Also given in Table 2 is the log of the scattering measure for a scattering medium assumed to have constant $C_{n}^{2}(x)$. The last column in Table 2 is the angular distance from Sgr A*. Seven of our 20 sources were not detected on any of the measured baselines but were seen in the autocorrelation spectra. For three of these sources we did not have data for our shortest baselines, because of a failure at PT, so it is perhaps not surprising that we did not detect these.

We do not give errors on the measurements for each source in Table 2. An accurate estimate of the uncertainties in the angular sizes is difficult to give, especially for the larger scattering sizes. They can generally not be estimated from the formal errors in the fit because the large angular sizes are often based on detections of a single baseline and are therefore a strong function of calibration and the detection threshold. Taking this into consideration, we estimate the errors on the large scatter sizes to be as high as $30 \%$. For the small scatter sizes we think we can rely upon the formal errors in the fit being on the order of $5 \%-10 \%$

\subsection{Known Lines of Sight}

To aid in our discussion we can enlarge the number of sampled lines of sight by including measured angular diameters from the literature. The four OH/IR stars of Van Langevelde \& Diamond (1991) were taken from the Lindqvist et al. (1992) sample and were observed with exactly the same telescope array and procedures as described above. We also use the measurements of $\mathrm{OH}$ and $\mathrm{H}_{2} \mathrm{O}$ masers in the Sgr B2 complex (Gwinn et al. 1988; Moran 1968). In addition, two radio transients have been discovered close to the Galactic center, both of which have had angular broadening measurements made over a range of wavelengths (Zhoa et al. 1991; Davies et al. 1976). Finally, of course, we include Sgr A* itself (Lo et al. 1985). For these last four sources it is known that their angular sizes scale quadratically with wavelength, consistent with their sizes being determined by interstellar scattering. The authors in each of the above-mentioned references also argue that their sources are located at the Galactic center. These extra eight lines of sight are presented in Table 3 in much the same format as Table 2.

\section{DISCUSSION}

$\mathrm{OH} 359.762+0.120$ was observed both here and by Van Langevelde \& Diamond (1991) and the $\theta_{\text {obs }}$ agree within the errors.

Figure 2 shows the distribution of scattering sizes in Galactic coordinates for the data taken from Tables 2 and 3. The sizes of the open circles are directly proportional to $\theta_{\text {obs }}$. The most striking aspect of this figure is the concentration of large scattering sizes near Sgr $A^{*}$. This is more evident in Figure 3 where we show the observed scatter size as a function of distance from Sgr $A^{*}$. There are seven measurements within $\pm 15^{\prime}$ of Sgr $A^{*}$ with an average $\theta_{\text {obs }}$ of 525 mas. In addition, we draw attention to the lower limits on $\theta_{\text {obs }}$ (upper limits on correlated flux) found on the left-hand side of Figure 3. These are the seven sources for which we failed to detect VLBI fringes. They are not randomly distributed about the range of sampled distances from Sgr A*, as would be expected if our nondetections were due to erors in pointing or poor sensitivity. It suggests that these lines of sight are more scattered still and that they are all but resolved by even our shortest baselines.

There are prominent variations in $\theta_{\text {obs }}$ in Figure 2 over short angular scales. Beyond the central region of $15^{\prime}$ radius, $\theta_{\text {obs }}$ drops precipitously to a value of 100 mas. Two sources are responsible for the drop, $\mathrm{Sgr} \mathrm{B} 2$ and $\mathrm{OH} 0.190+0.036$. In the past this result for Sgr B2 had been used to define the outer

TABLE 3

Additional Angular Broadening Measurements Scaled to $1612 \mathrm{MHz}$

\begin{tabular}{|c|c|c|c|c|c|c|}
\hline $\begin{array}{l}\text { Source } \\
\text { Name } \\
(1)\end{array}$ & $\begin{array}{c}l \\
(2)\end{array}$ & $\begin{array}{c}b \\
(3)\end{array}$ & $\begin{array}{c}\theta_{\text {obs }} \\
\text { (mas) } \\
(4)\end{array}$ & $\begin{array}{c}\log \mathrm{SM} \\
\left(\mathrm{kpc} \mathrm{\textrm {m } ^ { - 2 0 / 3 } )}\right. \\
(5)\end{array}$ & $\underset{(6)}{\Delta \operatorname{Sgr} A^{*}}$ & $\begin{array}{c}\text { References } \\
\text { (7) }\end{array}$ \\
\hline OH $359.762+0.120 \ldots \ldots$ & 395.762 & 0.120 & 531 & 2.51 & 14.8 & 1 \\
\hline OH $359.938-0.077 \ldots \ldots$ & 359.938 & -0.077 & 571 & 2.57 & 1.9 & 1 \\
\hline GCT $\ldots \ldots \ldots \ldots \ldots \ldots \ldots$ & 359.939 & -0.055 & 500 & 2.46 & 0.6 & 2 \\
\hline Sgr A* $A^{*} \ldots \ldots \ldots \ldots \ldots$ & 359.944 & -0.046 & 520 & 2.49 & 0.0 & 3 \\
\hline $\mathrm{A} 1742-28 \ldots \ldots \ldots \ldots \ldots$ & 359.930 & -0.043 & 530 & 2.51 & 0.9 & 4 \\
\hline OH $359.954-0.041 \ldots \ldots$ & 359.954 & -0.041 & 591 & 2.60 & 0.7 & 1 \\
\hline $\mathrm{OH} 0.190+0.036 \ldots \ldots \ldots$ & 0.190 & 0.036 & 103 & 1.08 & 15.6 & 1 \\
\hline Sgr B2 ................. & 0.7 & 0.01 & 96 & 0.98 & 45.5 & 5 \\
\hline
\end{tabular}

REFERENCES.- (1) van Langevelde \& Diamond 1991; (2) Zhao et al. 1991; (3) Lo et al. 1985; (4) Davies et al. 1976; (5) Moran 1968. 


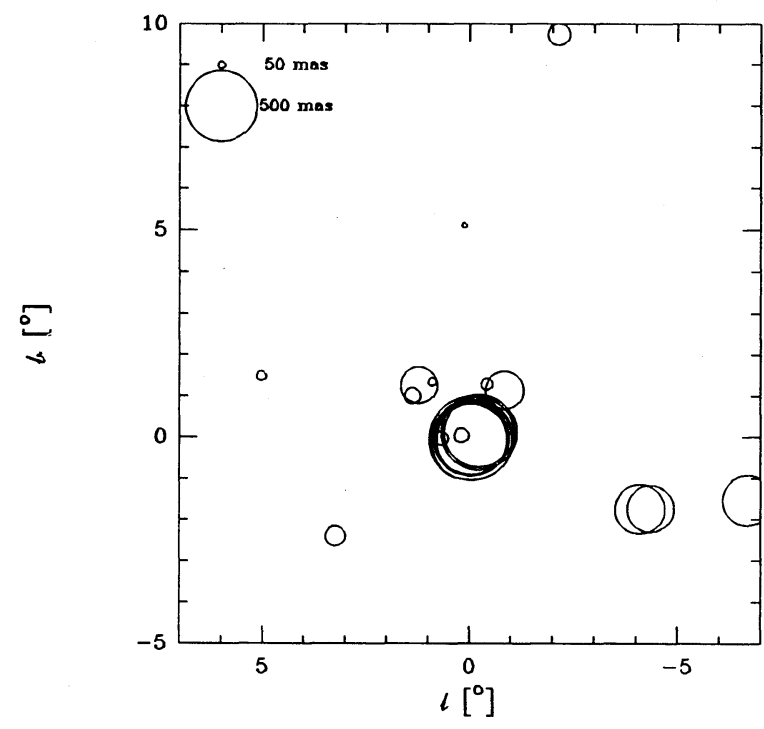

Fig. 2.-Scattering sizes for all the lines of sight near the Galactic center. The sizes of the circles indicate the amount of observed angular broadening.

limit for the size of the scattering region around $\mathrm{Sgr} \mathrm{A}^{*}$ to be $45^{\prime}$ or a maximum length scale of $150 \mathrm{pc}$ (Backer 1978; Ozernoi $\&$ Shishov 1977). With OH $0.190+0.036$ we can constrain the outer extent further still to $15^{\prime}$ or a radius of $40 \mathrm{pc}$. However, we caution that there are no IRAS colors for this star; thus the possibility exists that it is a foreground object. At $b \sim 1.5$ there are two additional examples of prominent variations taking place on short angular scales. Two pairs of sources, $\mathrm{OH}$ $359.140+1.137$ and $\mathrm{OH} 359.564+1.287$, and $\mathrm{OH} 1.212+1.258$ and $\mathrm{OH} 0.892+1.343$, are believed to be located at or beyond the Galactic center on the basis of their IRAS fluxes. In each pair the ratio of $\theta_{\text {obs }}$ varies by a factor of 3-5 over angular scales of $20^{\prime}-25^{\prime}$ which corresponds to order-of-magnitude variations in SM, over a maximum transverse distance of only $50-60 \mathrm{pc}$ (but an unknown distance along the line of sight). We also note that the data do not prove that scattering decreases monotonically from the Galactic center, because some of the lower bounds are more than a factor of 2 larger than actual

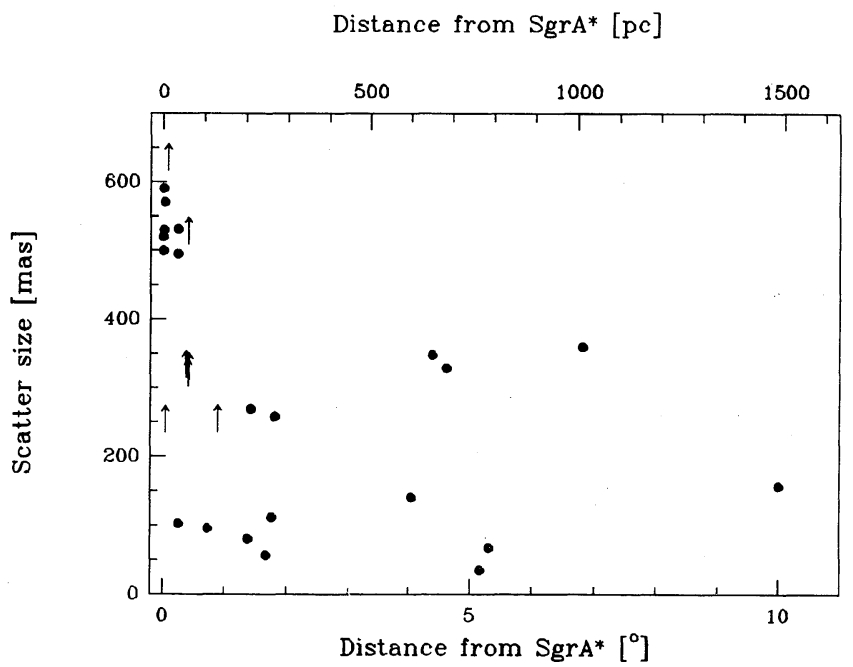

FIG. 3.-Plot of scatter sizes with distance from $\mathrm{Sgr} \mathrm{A}^{*}$ detections for similar angular distances from the Galactic center.

\subsection{Comparison with Other Scattering Measurements}

To put the Galactic center source measurements into context, we show in Figure 4 their scattering measures along with those of pulsars, other maser sources, and one active galactic nucleus (this figure is copied from Cordes et al. 1991a) for the area $-30^{\circ}<l<30^{\circ},-10^{\circ}<b<10^{\circ}$. We have calculated the SM for the Galactic center objects using equation (11) and assuming constant $C_{n}^{2}$. As can be seen, SM increases with distance much faster than would be expected if $C_{n}^{2}$ were constant and equal to the value inferred for the local interstellar medium. The rise occurs within the first few $\mathrm{kpc}$ from the Sun. The solid line in Figure 4 displays SM predicted from a best-fit model (Cordes et al. 1991a) to more than 200 lines of sight. The model consists of an outer Galaxy component that is nearly constant in galactocentric radius and has a $z$ scale height of 1 $\mathrm{kpc}$; an inner Galaxy annulus is centered on $R \approx 4 \mathrm{kpc}$, has a $e^{-1}$ width of $2 \mathrm{kpc}$, and a scale height of $100 \mathrm{pc}$. In terms of $C_{n}^{2}$, the inner Galaxy component is about 2000 times stronger than the outer component.

The model approximates SM data in other directions (e.g., toward the Galactic poles and toward the Galactic anticenter) with relatively little scatter. The large amount of scatter in Figure 4 is due to the small scale height $(100 \mathrm{pc})$ of the inner Galaxy component, corresponding to a Galactic latitude of only 0.7 at $8.5 \mathrm{kpc}$ distance. The dotted line shows the model's SM for $|l|=15^{\circ}, b=0^{\circ}$, while the dashed line shows the model looking out of the Galactic plane at $|b|=5^{\circ}$. Thus, points below the solid line may be understood as showing smaller SM because of the latitude dependence built into the model, while points above the line correspond to lines of sight with enhanced scattering (i.e., scattering in excess of that predicted by the model). For example, the masers inside NGC 6334 show enhanced scattering (as indicated) and the AGN behind it also shows enhanced scattering. The difference in SM for the background AGN and the maser is caused by the same leverage effect presented in equations (4) and (5).

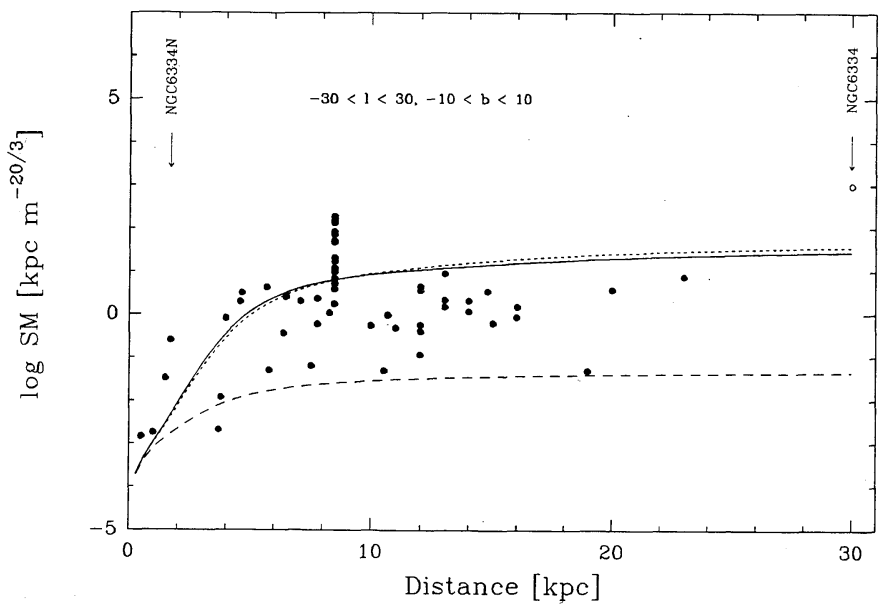

Fig. 4.-Plot of scattering measure against distance from the Sun. The plotted points represent data presented in Tables 2 and 3 and data compiled by Cordes et al. (1991a) that include pulsar measurements and data on scattered AGNs from Fey et al. (1991). AGNs are plotted arbitrarily at a "distance" of $30 \mathrm{kpc}$. The solid line represents SM calculated from the model of Cordes et al. for $l=0, b=0$; the dotted line corresponds to $|l|=15^{\circ}, b=0$; the dashed line corresponds to $l=0,|b|=5^{\circ}$. 
The most important feature of Figure 4 for the discussion here is the obvious enhancement of points for the sources with attributed distances of $8.5 \mathrm{kpc}$. The figure shows clearly that the scattering measures of the Galactic center sources are anomalous with respect to other lines of sight toward the inner Galaxy. We note, however, that the appearance of Figure 4, namely the sudden rise of SM from that of the model to about $10^{2.4} \mathrm{kpc} \mathrm{m}^{-20 / 3}$, does not prove that the enhanced scattering occurs at the distance of Sgr $\mathrm{A}^{*}$; none of the other sources plotted in the figure is at the same longitude and latitude, so the onset of the large enhancement could in fact occur, at a distance smaller than $8.5 \mathrm{kpc}$.

The line of sight toward Sgr A* and the $30^{\prime}$ diameter region around it does show an enhanced level of scattering, even when compared to lines of sight in the immediate vicinity. Where, then, does this turbulent medium originate and what can we say about its location relative to $\mathrm{Sgr} \mathrm{A}^{*}$ ? We consider two possible configurations to account for the turbulence: an anomalous scattering region close to $\mathrm{Sgr} \mathrm{A}^{*}$ and an unrelated turbulent region distributed in the interstellar medium toward Sgr A*, but not necessarily at the same distance as Sgr A*.

In the discussion we will adopt the formulas derived for very strong scattering, although the observations presented here do not yield any direct information on the presence of an inner scale. However, as pointed out in the Introduction, it seems likely that observations with $\theta_{\text {obs }}>100$ mas at $1 \mathrm{GHz}$ are affected by inner scale effects. Furthermore, the observed angular size of $\operatorname{Sgr} \mathrm{A}^{*} \propto \lambda^{2}$ seems to support this conclusion.

\subsection{Anomalous Scattering Local to the Galactic Center}

Ozernoi \& Shishov (1977) were the first to suggest that the scattering seen for Sgr A* occurred local to the source. They calculated $C_{n}^{2}$ for a single scattering screen located at several different positions along the line of sight, and then, assuming an outer scale $l_{0}$, they computed $\delta n_{e}$. They concluded that the ratio $\delta n_{e} / n_{e}$ was "reasonable" only when the scattering region was located 10 pc from Sgr A*. Backer (1978) independently concluded that the scattering towards $\mathrm{Sgr} \mathrm{A}^{*}$ was anomalous and gave the same distance of $10 \mathrm{pc}$ for the location of the scattering screen.

Our observations also may be interpreted in terms of a local origin for the scattering. As mentioned above, the zone of enhanced scattering appears to have a well-defined angular extent of $30^{\prime}$ approximately centered on $\mathrm{Sgr} \mathrm{A*}$, and there are no other lines of sight in our sample with comparable $\theta_{\text {obs }}$. There are strong phenomenological reasons for believing in a local origin, as the Galactic center is arguably one of the more energetic environments in our Galaxy.

We develop a single model to examine this hypothesis and use it to determine the physical properties of the scattering regions. In our model we assume there is an extended medium with $C_{n}^{2}(x)=C_{n, 0}^{2}$, and a region of enhanced scattering with $C_{n}^{2}(x)=C_{n, \mathrm{GC}}^{2}$ which is distributed in a sphere centered on $\mathrm{Sgr}$ $\mathrm{A}^{*}$ with a radius $R_{\mathrm{GC}}$. This model is evaluated using equations (8) and (10) and fitted to the data. The best-fit parameters are determined using a $\chi^{2}$ method. A distance of $8.5 \mathrm{kpc}$ is assumed for the distance of each source. This results in a fit of $C_{n, 0}^{2}=6.3$ $\mathrm{m}^{-20 / 3}, C_{n, \mathrm{GC}}^{2}=2.1 \times 10^{8} \mathrm{~m}^{-20 / 3}$ and $R_{\mathrm{GC}}=54 \mathrm{pc}$. This value for $C_{n, \mathrm{GC}}^{2}$ differs from that estimated by Van Langevelde \& Diamond (1991) for a similar geometry, because here we include inner scale effects $\left(l_{1}=100 \mathrm{~km}\right)$.

It is tempting to try to link the enhanced scattering to a known region at the Galactic center. In this respect we note that the size of $50 \mathrm{pc}$ coincides with the size of the "magnetosphere" of the Galactic center (Heyvaerts 1989).

\subsection{Anomalous Scattering due to Clumps Away from the Galactic Center}

It is well known that some lines of sight show enormously more scattering than neighboring ones, such as the Vela pulsar and Cygnus X-3. Moreover, the $\mathrm{H}$ II complex NGC 6334, at $l=351^{\circ}, b=0.7$, and $D \approx 1.7 \mathrm{kpc}$, causes scattering of a background extragalactic source that is larger than the scattering of the Galactic center sources discussed here (Moran et al. 1990). Cordes et al. (1985) discussed a model for the scattering medium which has a smoothly distributed component along with clumps of very strong turbulence. Clumps of parsec size were inferred to have a volume filling factor of about $10^{-4}$, corresponding to a mean free path for intersection of a clump of about 5-10 kpc. Using a more extensive data set, a model with two smooth components has been devised (Cordes et al 1991a, as shown in Fig. 4) that also must be augmented with clumps of turbulence with the same properties as the earlier model. Because the mean free path for intersecting a strong clump is less than or about equal to the distance to the Galactic center, it remains plausible that a similar clump may account for the enhanced scattering of the OH/IR masers and other sources in the Galactic center. Further observations of scattering in the inner Galaxy may indicate whether the line of sight is truly exceptional.

Previous authors have reported variations of scattering on angular scales of a few degrees or more (Fey, Spangler, \& Mutel 1989; Alurkar, Slee, \& Bobra 1986; Dennison et al. 1984). With our much finer grid of sampled points we are able to put an important limit on the typical size of a scattering clump, namely $\approx 50 \mathrm{pc}(D / 8.5 \mathrm{kpc})$, where $D$ is the distance of the clump from the Sun. The upper bound of $50 \mathrm{pc}$ is similar to clump sizes discussed by Cordes et al. (1985) and identical to that used by Fey et al. (1991) in their detailed model simulations.

\subsection{Implications and Predictions}

\subsubsection{Extragalactic Sources}

The model of enhanced scattering local to the Galactic center gives an interesting prediction for the observations of compact extragalactic sources, particularly those with flat spectra. Putting such a source behind the scatterer, within $15^{\prime}$ of $\mathrm{Sgr} \mathrm{A}^{*}$, we expect a size of $213^{\prime \prime}$ at $1.6 \mathrm{GHz}$. For a scattering medium close to Earth, this prediction comes out smaller, depending on the exact location of the scatterer. The best approach would be to make observations at higher frequencies (e.g., near $20 \mathrm{GHz}$ with the VLA) where the angular broadening is easier to measure (if the scattering arises in the vicinity of the Galactic center). Note that angular broadening decreases faster with an increase in frequency than does the angular resolution of a telescope.

\subsubsection{Physical Implications}

The location of the region that causes enhanced scattering of the Galactic center sources has a large influence on the amount of free-free absorption to be expected. Consider a clump model for the variations of $C_{n}^{2}$ with distance from the Galactic center:

$$
C_{n}^{2}(x)= \begin{cases}C_{n, 0}^{2}+C_{n, \mathrm{GC}}^{2} & \left|x-L_{\mathrm{GC}}\right| \leq \Delta L / 2 \\ C_{n, 0}^{2} & \text { otherwise }\end{cases}
$$


where $L_{\mathrm{GC}}$ is the distance of the clump from the Galactic center and $\Delta L$ is its thickness. Here, for simplicity, the value of $C_{n}^{2}$ outside the clump, $C_{n, 0}^{2}$, is assumed to be constant and much less than that inside the clump, $C_{n, G C}^{2}$. It may be shown, for $\Delta L / L \ll 1$, that

$$
\mathscr{S}(L) \approx \frac{1}{3} \mathrm{SM}_{0}+\mathrm{SM}_{\mathrm{GC}}\left(\frac{L_{\mathrm{GC}}}{L}\right)^{2}
$$

The total SM is

$$
\mathrm{SM}=\mathrm{SM}_{0}+\mathrm{SM}_{\mathrm{GC}} \approx\left(\frac{L}{L_{\mathrm{GC}}}\right)^{2} \mathscr{S}(L) .
$$

Introducing $\mathscr{S}(L)$ from equation (17) into equation (14) and using equation (11) we obtain

$$
\begin{aligned}
\tau_{\mathrm{ff}} \approx\left(\frac{L}{L_{\mathrm{GC}}}\right)^{2}\left(\frac{\theta_{\mathrm{obs}}}{7831.6 \mathrm{mas}}\right)^{2} & \left(\frac{l_{1}}{100 \mathrm{~km}}\right)^{1 / 3} \\
& \times\left(\frac{l_{0}}{1 \mathrm{pc}}\right)^{-2 / 3} \frac{v_{0, \mathrm{GHz}}^{4} g(v, T)}{v_{\mathrm{GHz}}^{2} T_{4}^{1.5}},
\end{aligned}
$$

where $v_{\theta, \mathrm{GHz}}$ is the frequency at which the angular broadening measurement $\theta_{\mathrm{obs}}$ is obtained and $v_{\mathrm{GHz}}$ is the frequency at which the optical depth is evaluated; $l_{1}$ is the inner scale, while $l_{0}$ is the outer scale. Using $\theta_{\text {obs }} \approx 500$ mas at $v_{\theta}=1.6 \mathrm{GHz}$ we obtain

$$
\tau_{\mathrm{ff}} \approx 0.027\left(\frac{L}{L_{\mathrm{GC}}}\right)^{2}\left(\frac{l_{1}}{100 \mathrm{~km}}\right)^{1 / 3}\left(\frac{l_{0}}{1 \mathrm{pc}}\right)^{-2 / 3} \frac{v_{\mathrm{GHz}}^{-2} g(v, T)}{T_{4}^{1.5}} .
$$

To have $\tau_{\mathrm{ff}} \leq 1$ at $1.6 \mathrm{GHz}$ requires that

$$
\frac{L_{\mathrm{GC}}}{L} \geq 0.10\left(\frac{l_{1}}{100 \mathrm{~km}}\right)^{1 / 6}\left(\frac{l_{0}}{1 \mathrm{pc}}\right)^{1 / 3}\left[\frac{g(v, T)}{T_{4}^{1.5}}\right]^{1 / 2} .
$$

It is possible to adjust the free parameters, particularly the temperature, to reduce the coefficient in equation (20) from 0.10 to 0.01 . It is also arguable that optical depth unity occurs well below $1.6 \mathrm{GHz}$, because the spectrum for Sgr $\mathrm{A}^{*}$ shows no break above $1 \mathrm{GHz}$ (Lo et al. 1985). However, there is no question that the optical depth is greater than unity at 327 $\mathrm{MHz}$, because Sgr $\mathrm{A}^{*}$ is absent in VLA images at that frequency (Anantharamaiah et al. 1991). Indeed, Pedlar et al. (1989) conclude that $\tau_{\mathrm{ff}}$ in front of Sgr $\mathrm{A}^{*}$ is $>2.5$ (at 327 $\mathrm{MHz}$ ), though it cannot be significantly larger than this value, otherwise the spectrum would turn over above $1 \mathrm{GHz}$. This would imply that

$$
\frac{L_{\mathrm{GC}}}{L} \leq 0.32\left(\frac{l_{1}}{100 \mathrm{~km}}\right)^{1 / 6}\left(\frac{l_{0}}{1 \mathrm{pc}}\right)^{1 / 3}\left[\frac{g(v, T)}{T_{4}^{1.5}}\right]^{1 / 2} .
$$

Therefore, observations of Sgr A* suggest that, if the scattering and absorbing material are one and the same, then (1) the outer and inner scales of the scattering medium must be nearly equal to the fiducial values of $1 \mathrm{pc}$ and $100 \mathrm{~km}$, respectively; and (2) that the scattering medium is situated quite far $(\geq 0.85 \mathrm{kpc})$ from the Galactic center.

\subsubsection{Implications for the Variability of $\mathrm{Sgr} A^{*}$}

The observations presented here give some idea about the electron density fluctuations close to Sgr $\mathrm{A}^{*}$. Even in the model in which the scattering medium is located in the vicinity of the
Galactic center, we find, from the distribution of scattered sizes, that it extends to 50 pc. A scatterer local to the Galactic center is also assumed in the discussion by Zhao et al. (1988). They infer that refractive scattering can explain the variability of $\mathrm{Sgr} \mathrm{A}^{*}$ if the medium is moving with a velocity $>1000 \mathrm{~km}$ $\mathrm{s}^{-1}$ relative to the source. Our observations make it unlikely that the diffractive scattering described in our paper and the refractive scattering postulated by Zhao et al. (1988) are caused by the same medium, because at a distance of $50 \mathrm{pc}$ from $\mathrm{Sgr}$ $A^{*}$ the velocities are reasonably well known and significantly below $1000 \mathrm{~km} \mathrm{~s}^{-1}$, although close to the position of Sgr A* high velocities have been reported (Geballe et al. 1991).

\subsubsection{Possible Associated Magnetic Irregularities}

Though the origin of electron density variations is unknown, it is plausible that they are related to, if not caused by, turbulence in the magnetic field such that

$$
\frac{\delta n_{e}}{n_{e}}=\zeta\left(\frac{\delta B}{B}\right)^{n}
$$

with $\zeta=1$ and $n=2$ for MHD turbulence and $n=1$ for obliquely propagating MHD waves (Cordes et al. 1990b). If $\delta n_{e} / n_{e}$ is of order unity, as it seems to be for lines of sight through the Cygnus region and for other Galactic plane lines of sight (e.g., Lazio, Spangler, \& Cordes 1990; Clegg et al. 1992), then the energy density in magnetic field irregularities (smaller than about $1 \mathrm{pc}$ ) is equal to the energy density in the large-scale field.

\section{CONCLUSIONS}

By simple detection experiments of $\mathrm{OH}$ masers around the Galactic center we show that lines of sight to the Galactic center are subject to severe interstellar scattering. We also show from changes in the scattering taking place on angular scales of $\approx 20^{\prime}$ that the upper limit on the size of a typical scattering clump is approximately $50 \mathrm{pc}$.

We consider two alternative interpretations for the location of the enhanced turbulence. Either it arises local to the Galactic center and is directly related to the violent processes going on within or it is due to a clump of enhanced turbulence along the line of sight and has no physical relationship to the Galactic center. Our data can be fitted to either model equally well, but the former model seems to conflict with observations of freefree absorption toward Sgr $A^{*}$, in which the same free electrons should play a role in scattering. Further observations are suggested to help resolve this controversy.

We thank the VLBA operations team for making the observations and their processing possible, especially Joan Wrobel, Craig Walker, Barry Clark, and Clint Janes. Harm Habing is thanked for his continuous advice. We are indebted to Anders Winnberg and Michael Lindqvist for letting us use their catalog prior to publication. H. J. vL thanks the NRAO for their hospitality, and likewise D. A. F. thanks Sterrewacht Leiden.

D. A. F. acknowledges the financial support of the Natural Sciences and Engineering Research Council of Canada through an NSERC Postdoctoral Fellowship and the National Radio Astronomy Observatory through a Jansky Fellowship.

J. M. C. acknowledges NASA grant NAGW-1646 for support of this research along with support from the National Astronomy and Ionosphere Center which operates Arecibo Observatory under contract with the NSF. 


\section{REFERENCES}

Alcock, C., \& Ross, R. R. 1986, ApJ, 305, 837

Alurkar, S. K., Slee, O. B., \& Bobra, A. D. 1986, Australian J. Phys., 39, 433

Anantharamaiah, K. R., Pedlar, A., Ekers, R. D., \& Goss W. M. 1991, MNRAS, submitted

I Armstrong, J. W., Cordes, J. M., \& Rickett, B. J. 1981, Nature, 291, 561

Backer, D. C. 1978, ApJ, 222, L9

. 1988, in Radio Wave Scattering in the Interstellar Medium, ed. J. M. Cordes, B. J. Rickett \& D. C. Backer (New York: AIP), 111

Benson, J. M., \& Mutel, R. L. 1979, ApJ, 233, 119

Blommaert, J. A. D. L., van Langevelde, H. J., van der Veen, W. E. C. J., Habing, H. J., \& Epchtein, N. 1992, in preparation

Bowers, P. F., Reid, M. J., Johnston, K. J., Spencer, J. H., \& Moran, J. M. 1980, ApJ, 242, 1088

Boyd, R. B., \& Werner, M. W. 1972, ApJ, 174, L137

Burke, B. F., Moran, J. M., Barett, A. H., Rydbeck, O., Hansson, B., Rogers, A. E. E., Ball, J. A., \& Cudaback, D. D. 1968, AJ, 73, S168

Clegg, A. W., Cordes, J. M., Simonetti, J. H., \& Kulkarni, S. R. 1992, ApJ, 386, 143

Clifton, T. R., \& Lyne, A. G. 1986, Nature, 320, 43

Cordes, J. M., Anathakrishnan, S., \& Dennison, B. 1984, Nature, 309, 689

Cordes, J. M., Clegg, A., \& Simonetti, J. 1990a, in IAU Symp. 140, Galactic and Intergalactic Magnetic Fields, ed. R. Beck, P. P. Kronberg, \& R. Wielebinski (Dordrecht: Kluwer), 55

Cordes, J. M., \& Lazio, T. J. 1991, ApJ, 376, 123

Cordes, J. M., Spangler, S. R., Weisberg, J. M., \& Ryan, M. 1991a, ApJ, submitted

Cordes, J. M., Weisberg, J. M., \& Boriakoff, V. 1985, ApJ, 288, 221

Cordes, J. M., Weisberg, J. M., Frail, D. A., Spangler, S. R., \& Ryan, M. 1991b, Nature, submitted

Cordes, J. M., Wolszczan, A., Dewey, R., Blaskiewicz, M., \& Stinebring, D. R. 1990b, ApJ, 349, 245

Davies, R. D., Walsh, D., \& Booth, R. S. 1976, MNRAS, 177, 319

Dennison, B., Thomas, M., Booth, R. S., Brown, R. L., Broderick, J. J., \& Condon, J. J. 1984, A\&A, 135, 199

Diamond, P. J. 1989, in Very Long Baseline Interferometry, Techniques and Applications, ed. M. Felli \& R. F. Spencer (Dordrecht: Kluwer), 231

Diamond, P. J., Martinson, A., Dennison, B., Booth, R. S., \& Winnberg, A. 1988, in Radio Wave Scattering in the Interstellar Medium, ed. J. M. Cordes, B. J. Rickett, \& D. C. Backer (New York: AIP), 195

Dickinson, D. F. 1987, ApJ, 313, 408

Fey, A. L., Spangler, S. R., \& Cordes, J. M. 1991, ApJ, 372, 132

Fey, A. L., Spangler, S. R., \& Mutel, R. L. 1989, ApJ, 337, 730

Gaylard, M. J., West, M. E., Whitelock, P. A., \& Cohen, R. J. 1989, MNRAS, 236,247
Geballe, T. R., Krisciunas, K., Bailey, J. A., \& Wade, R. 1991, ApJ, 370, L73

Gwinn, C. R., Moran, J. M., Reid, M. J., \& Schneps, M. H. 1988, ApJ, 330, 817

Heyvaerts, J. 1989, in IAU Symp. 136, The Center of the Galaxy, ed. M. Morris (Dordrecht: Kluwer), 301

Ishimaru, A. 1978, Wave Propagation and Scattering in Random Media (New York: Academic)

Kemball, A., Diamond, P. J., \& Mantovani, F. 1988, MNRAS, 234, 713

Kent, S. R., \& Mutel, R. L. 1982, ApJ, 263, 145

Lazio, T. J., Spangler, S. R., \& Cordes, J. M. 1990, ApJ, 363, 51

Lindqvist, M., Winnberg, A., Habing, H. J., \& Matthews, H. E. 1992, A\&AS, in press

Lo, K. Y., Backer, D. C. Ekers, R. D., Kellermann, K. I., Reid, M., \& Moran, M. J. 1985, Nature, 315,124

Lo, K. Y., Cohen, M. H., Readhead, A. C. S., \& Backer, D. C. 1981, ApJ, 249, 508

Molnar, L., Mutel, R., Reid, M., \& Johnston, K. 1990, ApJ, submitted

Moran, J. M. 1968, Ph.D. thesis, MIT

Moran, J. M., et al. 1973, ApJ, 185, 535

Moran, J. M., Rodriguez, L. F., Greene, B., \& Backer, D. C. 1990, ApJ, 348, 150

Norris, R. P., Booth, R. S., Diamond, P. J., Nyman, L. A., Graham, D. A., \& Matveyenko, L. I. 1984, MNRAS, 208, 435

Ozernoi, L. M, \& Shishov, V. I 1977, Soviet Astron. Lett, 3, 233

Pedlar, A., Anantharamaiah, K. R., Ekers, R. D., Goss, W. M., Van Gorkom, J. H., Schwarz, U. J., \& Zhao, J. 1989, ApJ, 342, 769

Rao, A. P., \& Slee, O. B. 1988, MNRAS, 234, 853

Reid, M. J., Muhleman, D. O., Moran, J. M., Johnston, K. J., \& Schwartz, P. R. 1977, ApJ, 214, 60

Rickett, B. J. 1990, ARA\&A, 28, 56

Röttgering, H. J. A. 1989, A\&A, 222, 125

Spangler, S. R., \& Gwinn, C. R. 1990, ApJ, 353, L29

te Lintel Hekkert, P., Caswell, J. L., Habing, H. J., Haynes, R. F., \& Norris, R. P. 1991, A\&AS, in press

Van der Veen, W. E. C. J., \& Breukers, R. J. L. H. 1989, A\&A, 213, 133

Van Langevelde, H. J., \& Diamond, P. J. 1991, MNRAS, 249, 7P

Van Langevelde, H. J., Van der Heiden, R., \& Van Schooneveld, C. 1990, A\&A 239,193

Wilson W. J \& Barett, A. H. 1968 Science, 161,778

Zhao, J. H., Ekers, R. D., Goss, W. M., Lo, K. Y., \& Narayan, R. 1988, in IAU Symp. 136, The Center of the Galaxy, ed. M. Morris (Dordrecht: Kluwer), 535

Zhao, J. H., Roberts, R. A., Goss, W. M., Frail, D. A., Lo, K. Y., Subrahmanyan, R., Ekers, R. D., \& Kesteven, M. J. 1991, Science, submitted 